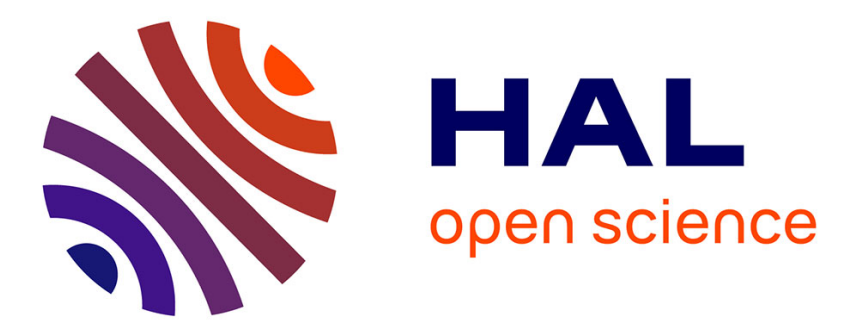

\title{
Pricing Strategies in Multi-Operator Heterogeneous Wireless Networks
}

\author{
Soha Farhat, Abed Ellatif Samhat, Samer Lahoud, Bernard Cousin
}

\section{To cite this version:}

Soha Farhat, Abed Ellatif Samhat, Samer Lahoud, Bernard Cousin. Pricing Strategies in MultiOperator Heterogeneous Wireless Networks. 7th IFIP International Conference on New Technologies, Mobility and Security, Jul 2015, Paris, France. 10.1109/NTMS.2015.7266513 . hal-01155538

\section{HAL Id: hal-01155538 \\ https://hal.science/hal-01155538}

Submitted on 28 May 2015

HAL is a multi-disciplinary open access archive for the deposit and dissemination of scientific research documents, whether they are published or not. The documents may come from teaching and research institutions in France or abroad, or from public or private research centers.
L'archive ouverte pluridisciplinaire $\mathbf{H A L}$, est destinée au dépôt et à la diffusion de documents scientifiques de niveau recherche, publiés ou non, émanant des établissements d'enseignement et de recherche français ou étrangers, des laboratoires publics ou privés. 


\title{
Pricing Strategies in Multi-Operator Heterogeneous Wireless Networks
}

\author{
Soha Farhat ${ }^{1,2}$, Abed Ellatif Samhat ${ }^{1}$, Samer Lahoud ${ }^{2}$, Bernard Cousin ${ }^{2}$ \\ soha.farhat@ul.edu.lb,samhat@ul.edu.lb, samer.lahoud@irisa.fr, Bernard.Cousin@irisa.fr \\ ${ }^{1}$ Lebanese University, Faculty of Engineering-CRSI,Hadath, Lebanon \\ ${ }^{2}$ University of Rennes I - IRISA, France
}

\begin{abstract}
In this paper, three pricing scenarios are proposed to set the transaction cost of the inter-operators agreement in a multi-operator cooperative environment. An analysis, of the operators' profits, is performed for these cooperation scenarios and different price sharing models are investigated for comparison. First, we describe the proposed pricing scenarios, the motivation behind and the points of evaluation for each scenario. Then, we present the hybrid decision algorithm for the selection of the access in a multi-operator wireless networks environment. Next, we present two business modelsmade for the simulation, in order to highlight how network conditions and operator's strategy for service price may affect the profitability of the cooperation. Simulation results show that proposed pricing models guarantee profit gain for the cooperating operators, and are suitable in a multi-operator sharing environment. A best pricing scenario can be decided depending on the deployed capacity for sharing and the user's service price settings.
\end{abstract}

Keywords-Multi-operator sharing networks; cooperation; access selection; service pricing; transaction cost, cooperation awareness.

\section{INTRODUCTION}

The mobile broadband traffic is growing in a rapid manner, facing network operators with the challenge of expanding capacity and enhancing the Quality of Service (QoS) of their communication networks. In addition, operators are worried about the decrease of revenues especially from voice services, and they sense the need of new technical and network solutions that can generate new sources of revenues. Business solutions introduced in [1]show that mobile operators cooperate with other competitors and customers; some proposed partners can be providers of a non-telecom service like financial institutes, public transportation or third parties taking intermediary roles. Multi-operators cooperation in the form of open access and always best connected, has been proposed in a number of large research projects likeAmbient Networks[2], SPICE[3]... etc. and several types of cooperation between networks and business entities are made. The cooperation of wireless network operators in a sharing environment is also introduced in [3] as a cost effective network solution to expand capacity and improve operators' profitability. Indeed, many works has showed that in a heterogeneous wireless environment, operators' cooperation is unavoidable and inter-operator agreements can bring benefits in terms of both network performance and operators' revenues [5][6]. In addition, new mobile architecture arises in order to help operator upgrading their access networks, and enable network sharing in a Cloud Radio Access Network [7].

In our work, we consider a cooperative environment where wireless network operators share their access resources, to upgrade their networks' capacity and improve performance in terms of clients' rejection and QoS degradation. In such cooperative environment, when an operator is unable to satisfy his client, he gives him access to the service through another network operator, thus avoiding his rejection. Consequently, a selection decision is needed when more than one operator are available for cooperation. In addition, a transaction cost is to be set in order to guarantee QoS and achieve additional revenues thus, making cooperation more profitable.

In the context of multiple operators, most of the recent works study the selection of access and service pricing using game theory as in [9][10]. In a previous work, we proposed a cost function for the selection decision in a multi-operators environment, and we showed the efficiency of our algorithm in [11] and how the operator can control the selection decision in [12].

In this paper, three new pricing scenarios are proposed as flat price scenarios for the inter-operator transaction cost. We perform a thorough analysis of these pricing scenarios and a comparison with price sharing scenarios. Moreover, the proposed scenarios for a flat transaction cost price presented better profitability comparing to price sharing models, in some operator cases of deployed capacity and service price settings.

The rest of the paper is organized as follows: Section II presents relevant existing work related to business models in a multi-operator environment. Section III describes the proposed pricing scenarios for the transaction cost in a sharing environment, andthedecision algorithm used for selectionis presented in section IV. The simulated business models are depicted in section V. Section VI shows the simulation results for the efficiency of the selection algorithm, and elaborates the results for the pricing scenarios profitability, in each business model. Finally, in section VII, a conclusion is made for the best pricing scenario in function of capacity and service price settings.

\section{BACKGROUNG AND RELATED WORKS}

Mobile operator cooperation is introduced in [1] as a networking solution to reduce networks cost and generates new type of revenues. The author focuses on cost saving 
strategies based on cooperation through network sharing, spectrum sharing and roaming, besides femtocell deployment. Investigations on the drivers of cooperation revealed that from cost perspective the incentive to share networks might be lower today since a large number of base station sites can be re-used and since the price of radio equipments has been reduced. But, entering to the mobile market and keeping a position in this market motivate mobile competitors to cooperate[13].

When cooperation decision is made, the sharing partners have to agree on several aspects such as: how much of the network should be shared, how to share costs for investment and use of the network, how to make decision for network expansion, etc. Thus, a sharing agreement must be settled between different competitors in order to manage radio resources in such multi-operator, multi-access, wireless networks. In [14], authors describe the business models for shared networks, based on fragmented wireless access and service market. Two examples are presented, the first includes Mobile Network Operators (MNOs) that offer wide area wireless access to specialized service providers, and the second includes Local Service and Access Providers (LSAPs), interactingwith service providers and mobile operators via Inter-Connection Provider (ICP). In such a fragmented market, an LSAP may also provide services via other LSAPs or MNOs networks, and MNOs can also lease capacity from LSAPS, thus a cooperative environment can be envisaged. Moreover, authors propose that network selection is performed by either the service provider or an ICP to preserve competition and reduce transaction costs. This ICP will also maintain the Service Level Agreement between radio access providers and service providers. The concept of a third trusty party is adopted also in [5] for inter-operators joint resource management. Inter-operators agreements for network selection decision and users' transaction cost are maintained and guaranteed by a meta-operator acting as a trading agent between cooperating operators.

Authors propose in [5] a two-layer JRRM (Joint Radio Resource Management) strategy based on fuzzy neural methodology. Its objective is to provide the most appropriate RAT (Radio Access Technology). Then, authors assumed that the total revenue generated by the user $p$ is shared between the two involved operators. Thisenables the service operator to get $\alpha p$ where $0 \leq \alpha \leq 1$. The first model consists of an interoperator agreement where the service operator gets all revenue with $\alpha=1$, this model is more beneficial for the operator that correctly estimates the infrastructure deployment. The second model consists to share revenue in function of the normalized load $\eta$ of the service operator with $\alpha=\eta$, this model is fair for the service operator. A second work of the authors [6]proposes an additional revenue sharing model based on the service quality experienced by the users in terms of churning rate of the home operator which is a function of its blocking probability $P_{b}$, thus setting $\alpha=C\left(P_{b}\right)$ the user's churning rate. Performance evaluation showed that this novel sharing model keeps a fairer behavior of both previously proposed business models.
In this paper, we propose three new pricing models for the inter-operator transaction cost. The transaction of a user consists of transferring this user from his home operator $(\mathrm{H}-$ op) which has contract with, to a new service operator (S-op). The latter sets the transaction cost $C s$ and the H-op will pay it. The global achieved profits are compared using these new models and the price sharing models, in order to conclude the best pricing scenario for an operator qualified as the cheapest operator or having the best capacity deployment.

\section{TRANSACTION COST PRICING}

The inter-operator financial agreement should determine how the user transaction cost $C s$ is set between cooperating operators. In this paper, we test the profitability of three new pricing scenarios, where $\mathrm{S}-\mathrm{op}_{\mathrm{i}}$ sets $C s_{i}$, by three different functions of the service price $p_{i}$ asdescribed in the following $\left(p_{i}\right.$ is the price paid by the client of the operator $i$ ).

\section{A. ScenarioS1:}

To prevent any loss of investment, a guest user (user coming from another operator)must generate the same revenue as from a client user. Thus, the transaction cost of a S-op is set equal to the service price, thus $C s_{i}=p_{i}$. In this scenario, we intend to assess the following points:

1. Profits improvement especially for operators with cheap service price $s p$.

2. Benefit from cooperation of an operator with the most expensive service cost.

3. Effect on the client acceptance especially for operators with the cheapest $s p$.

\section{B. Scenario $S 2$ :}

We may notice, in $S 1$, that an operator having the cheapest service price will pay a high price for its client transfer and gain less from guest users. It could face losses when client transaction is frequent. Thus, in this scenario, we propose that $C s=\max _{i}\left(s p_{i}\right) i=1,2,3 \ldots$ In this scenario, it is guaranteed that all available S-op offers set the same cost for H-op. Hence, we intend to test if:

1. The cooperation still beneficial for operators even when it causes profit losses.

2. The operator having the cheapest price is improving his profits.

\section{Scenario S3:}

To improve users' acceptance, an operator may perform a high rate of user's transaction, which causes a lot of charges in $\mathrm{S} 2$. Operators may find better to pay less and get less than pay more. Thus, $\mathrm{S} 3$ proposes a price $C s=\min _{i}\left(s p_{i}\right) i=1,2,3 \ldots$ The study of this scenario targets the possibility of achieving profit gain with a low service cost.

\section{Scenario pShare:}

With price sharing $\mathrm{S}$-op takes a share from the user payment $\alpha p$ thus, H-op keeps $(1-\alpha) p$, where $\alpha \geq 0$. Depending on the value of $\alpha$, different sub-scenarios can be envisaged: 
1) Scenario pShare 1: In this model, $\alpha=1$, i.e, S-op gets all the revenue from user transfer.

2) Scenario pShare0: In this model, $\alpha=0$, i.e, no charges are depicted for user exchange, and H-op gets all client's payment.

3) Scenario pShare-: In this model, $\alpha<1$, i.e, additional revenues are guaranted for both $\mathrm{H}-\mathrm{p}$ and S-op. Without loss of generality we show the results for $\alpha=0.25$ and 0.6 .

\section{OPERATOR SELECTION DECISION}

In our previous work [11], we proposed a cost function $C F$ thatenables to select the operator having a score $S_{T}$ at minimum distance of the user score $S u$, while maximizing the home operator transaction profit $(p-C s)$. Simple Additive Weighting SAW is used to calculate $S u$ which combines the QoS requirements of the user application, and $S_{T}$ which combines corresponding QoS parameters delivered by the Sop. Thus, the selected $S$-opi is the operator having the lowest $C F$, with:

$$
C F_{i}=W_{u} *\left|S u-S_{T i}\right|-W_{o p} *\left(p-C s_{i}\right)
$$

Where, $W u$ and Wop are weighting coefficients that determine the degree of importance for the user satisfaction compared to profit satisfaction, respectively. Details concerning the cost function $C F$ are provided in our previous work [11].

\section{SIMULATED SYSTEM MODELS}

For the simulations, we consider two business models $M I$, and $M I I$.

\section{A. MI-Capacity effect}

In model MI, we assume that all operators deliver the same QoS specifications for the mobile users and set the same service price $s p_{1}=s p_{2}=s p_{3}$, then we consider different capacity for each operator. Setting the same service price for all operators reduces the simulations to $S 1$ and pShare scenarios.

\section{B. MII-Service Price effect}

In model MII, we assume that all operators deliver the same QoS specifications, have the same capacity, but set different service prices $s p$.

These business models are made for simulation, in order to reveal which pricing scenario is more profitable in function of the shared capacity and the service price.

\section{SIMULATION RESULTS}

\section{A. Simulation Setup}

We consider three cooperating operators; $O p_{1}, O p_{2}$ and $O p_{3}$ as in Fig.1, The delivered parameters and the service prices for each operator are depicted in Table I, for each model MI and MII.

Users arrive to the system sequentially and we model the arrival and departure of users as a Poisson Process with mean arrival interval $1 / \lambda$ seconds. We perform simulation for different values taken from $1 / \lambda=\left[\begin{array}{llllll}6 & 4.8 & 4 & 3.43 & 3 & 2.67\end{array}\right]$. Once

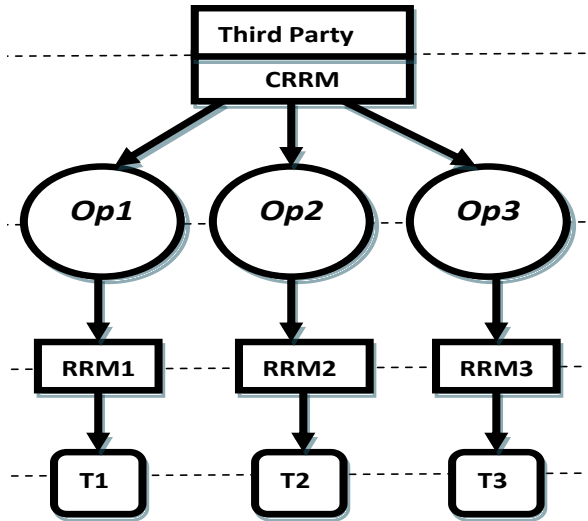

Fig.1.Multi-operator system model

connected, the user will stay in the system for a service time assumed to follow an exponential distribution of mean $1 / \mu=4 \mathrm{~min}$. The simulations are implemented in Matlab for duration of 1200 seconds each and repeated for 30 experiments.

TABLE I. OPERATORS' NETWORK PARAMETERS

\begin{tabular}{|c|c|c|c|}
\hline \multirow[b]{2}{*}{$\begin{array}{l}\text { System } \\
\text { model }\end{array}$} & \multicolumn{3}{|c|}{ Operators' Network Settings } \\
\hline & $\begin{array}{l}\text { Capacity }(\mathrm{Kb} / \mathrm{s}) \\
{\left[\mathrm{Op}_{1}, \mathrm{Op}_{2}, \mathrm{Op}_{3}\right]}\end{array}$ & $\begin{array}{c}\text { Service } \\
\text { Price(units/Kbytes) }\end{array}$ & $\begin{array}{l}\text { QoS } \\
\text { specifications } \\
{\left[J_{M}, D_{M}, B_{E} R_{M}\right]}\end{array}$ \\
\hline MI & $\begin{array}{c}{[11000,9000} \\
5000]\end{array}$ & $s p=0.5$ & {$\left[10,30,10^{-5}\right]$} \\
\hline MII & 11000 & $\begin{array}{c}s p_{1}=0.3, s p_{2}=0.5 \\
\quad \text { and } s p_{3}=0.9\end{array}$ & {$\left[10,30,10^{-5}\right]$} \\
\hline
\end{tabular}

\section{B. Selection Algorithm efficiency}

The efficiency of the selection algorithm is shown through the blocking rate improvement for each operator of the system $(O p 1, O p 2$, and $O p 3)$, and the profits achieved via cooperation. The comparison of performance in terms of blocking rate and profits is done for the scenarios $S 1, S 2, S 3$ and the case where there are no inter-operator agreements for cooperation.

\section{1) Blocking rates improvement}

Figures 2a, 2b and 2cshow how the cooperation between $O p 1, O p 2$, and $O p 3$, respectively,could reduce the blocking rates especially for high number of system arrivals. The maximum number of admitted users for each operator is increased of more than $20 \%$, inducing an increase of the user acceptance in the whole system up to $24 \%$ for a blocking probability of $2 \%$. This translates the capacity gain achieved through cooperation. Note that this improvement is the same for the three inter-operator agreements $S 1, S 2$ and $S 3$.

\section{2) Profits Improvement}

Figures $3 \mathrm{a}, 3 \mathrm{~b}$ and $3 \mathrm{c}$ show the total profits achieved by $O p 1, O p 2$ and $O p 3$, respectively. Operators could gain more revenues through cooperation; the user acceptance is improved and extra revenues are gained from guest users. The proposed pricing scenarios guaranteed higher profits for all 


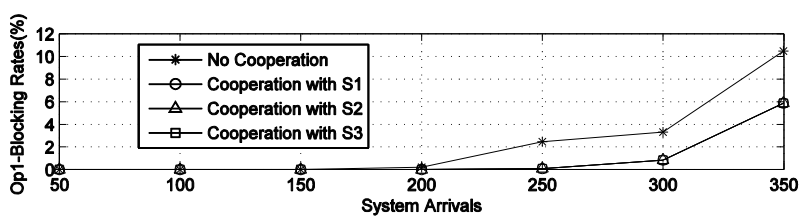

Fig. 2a.Op1's blocking rates improvement

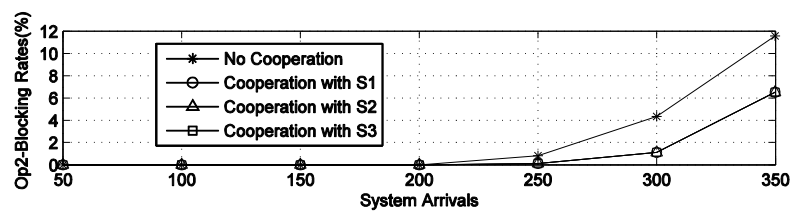

Fig. 2b.Op2's blocking rates improvement

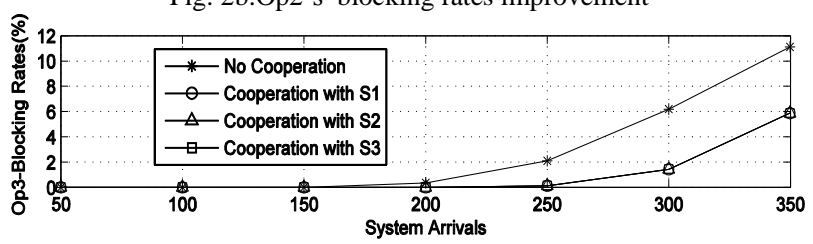

Fig. 2c. Op3's blocking rates improvement

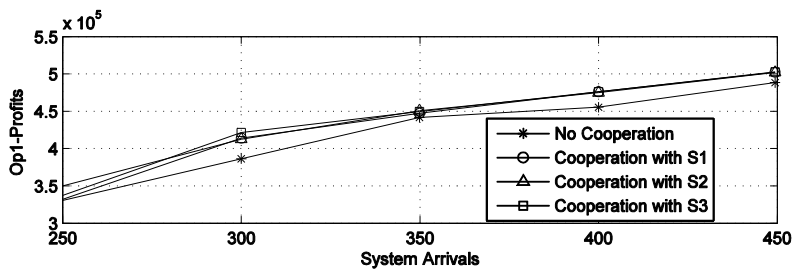

Fig. 3a. Op1's profit improvement

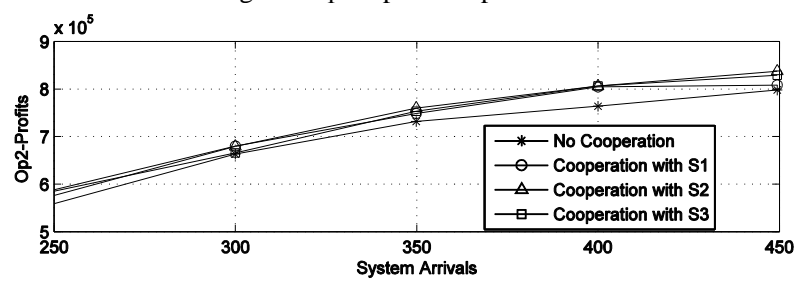

Fig. 3b. Op2's profit improvement

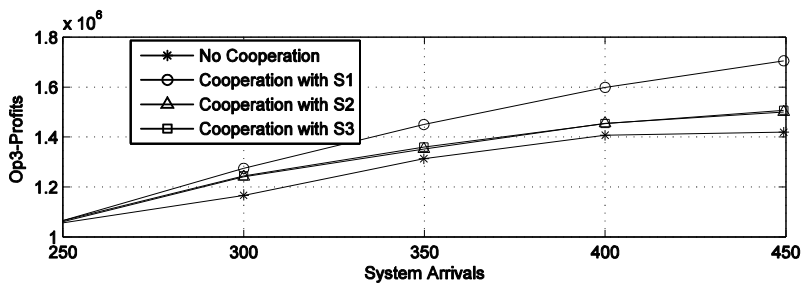

Fig. 3c.Op3's profit improvement

cooperating operators. In addition, Fig. $3 \mathrm{c}$ shows that $S 1$ is the best pricing scenario to adopt by $O p 3$, which sets a high service price $p$.

\section{Pricing Scenarios Comparison}

\section{1) Capacity based Comparison-Model MI:}

In model MI, operators differ in the deployed capacity for sharing Table I. Figures $4 \mathrm{a}, 4 \mathrm{~b}$ and $4 \mathrm{c}$ show the profit achievements for $O p 1, O p 2$ and $O p 3$, respectively, with each pricing scenario in addition to the case where no inter-operator agreement is made (No cooperation scenario).

First, note that $S 1, S 2$ and $S 3$ produce the same profits as the pSharel scenario, for all operators $\left(p_{1}=p_{2}=p_{3}\right)$. The

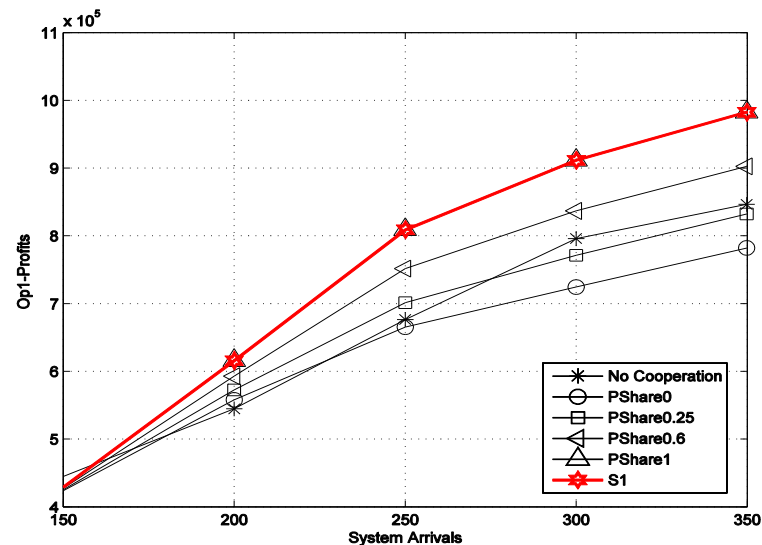

Fig. 4a. Op1's achieved profit (with high capacity)

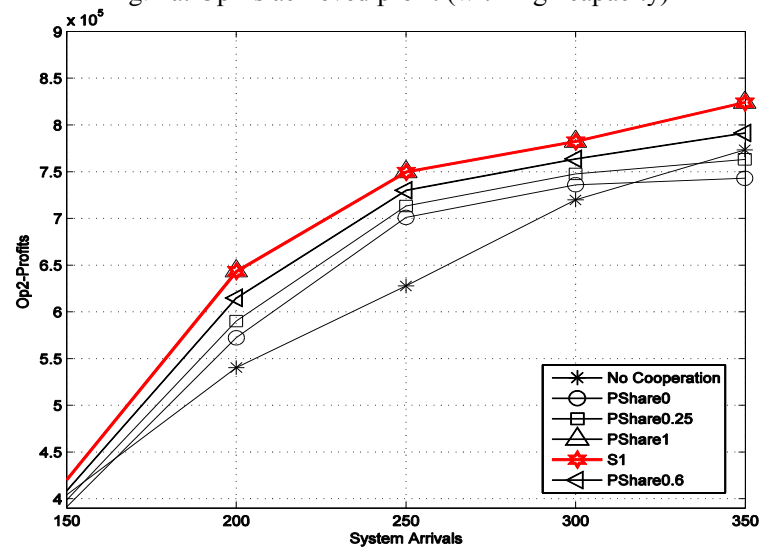

Fig. 4b. Op2's achieved profit (with moderate capacity)

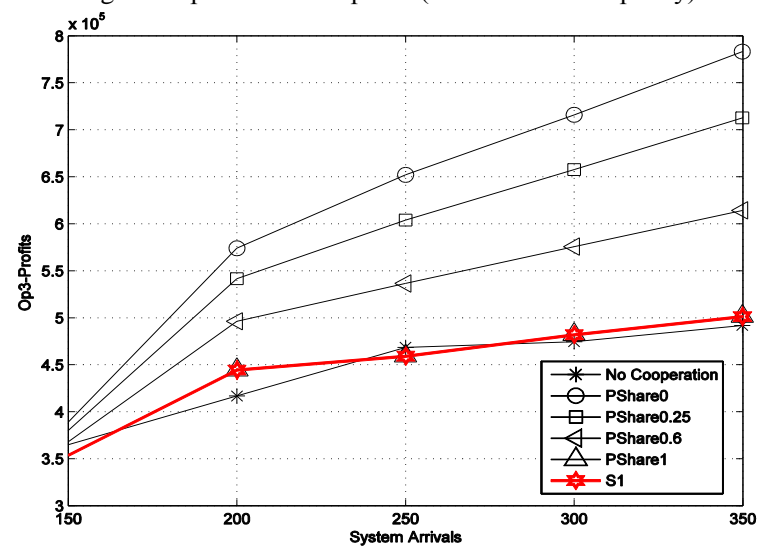

Fig. 4c. Op3's achieved profit (with low capacity)

proposed scenarios guarantee high profits for the operator deploying a high capacity. But, these scenarios do not improve the achieved profits for $O p 3$, having the lowest capacity, and losses may occur at same number of system arrivals. The proposed pricing scenarioS1,retaining the same price for clients and cooperating operators $C s_{i}=p_{i}$, guaranteesthe highest transaction cost (0.5 units/Kbytes) for the S-op compared to the pShare scenarios (with $\alpha<1$ ). Thus, as much the cooperating operator can serve guest users as much it gets profits. However, when the operator wants to improve its user acceptance with a lot of client transfer to another S-op, high charges have to be paid. In addition, with the pShareO scenario, where the H-op keeps all its client payment and S-op 
loses additional revenues from guest user. This scenario causes a lot of losses for $O p 1$, at high system arrivals. In fact, at these rates, $O p 1$ is serving a high number of guest users, without additional revenues that may recover charges or probable client payments. Thus, our proposed pricing scenarios guarantee the best profits for the operators having a good dimensioning for sharing.

\section{2) Service price based Comparison-Model MII:}

In model MII, operators differ in the service prices $\left(s p_{1}<s p_{2}<s p_{3}\right)$. We are interested to show the profit improvement for $O p 1$ and $O p 3$ setting the cheapest and the most expensive service price $p$, respectively.

Figure $5 \mathrm{a}$ and $5 \mathrm{~b}$ show the profits achieved by $O p l$ and $O p 3$, respectively, with the different pricing scenarios. Results show that with the proposed pricing scenarios $S 1, S 2$ and $S 3$, Op3 could maximize its profits especially for high system arrivals, where other price sharing scenarios cause losses. In fact, $O p 3$ could achieve the highest profits with S1. For $O p 1, S 2$ and $S 3$ could improve its profits via cooperation but not as much price sharing scenarios did. Scenario $S 1$ causes losses for $O p 1$, at high system arrivals, where this operator transfers its clients to more expensive operators, and served guests do not assure enough revenues to recover transaction cost. Hence, $S 1$ is to be avoided by the operator setting the lowest service price.

\section{CONCLUSION}

In this paper, three pricing scenarios are proposed for the transaction cost of the inter-operator agreement, in order to improve operators' revenues through cooperation, in a multioperator environment. These scenarios are analyzed and compared togetherand with different price sharing scenarios.

Simulation results, using our modified cost function for access selection decision, have shown that the establishment of inter-operator agreements brings benefits in terms of user acceptance and operators' profits. The proposed scenario $S 1$, that maintain the same price for clients and cooperating operators has been shown as the best financial agreement for the operators having a good dimensioning for shared capacity, and the operator setting the higher service price in the cooperating system. When Slcaused losses for the operator setting the lowest service price in the system, other scenarios $S 2$ and $S 3$ could improve profits and make cooperation profitable. Future work will take advantage of game theory as a tool for operator selection and inter-operator service pricing.

\section{REFERENCES}

[1] J. Markendahl, Mobile Network Operators and Cooperation: PhD thesis in telecommunication, Stockholm 2011,chap.3 pp.31-80.

[2] Norbert Niebert, Hannu Flinck, Robert Hancock, Holger Karl and Christian Prehofer. "Ambient Networks - Research for Communication Networks Beyond 3G”. Proc. IST Mobile Summit, June 2004.

[3] S. Tarkoma, B. Bhushan, E. Kovacs, H. van Kranenburg, E. Postmann, R. Seidl, and A. V. Zhdanova, "Spice: A service platform for future mobile ims services," in World of Wireless, Mobile and Multimedia Networks, 2007. WoWMoM 2007. IEEE International Symposium on a, June 2007, pp. 1-8.

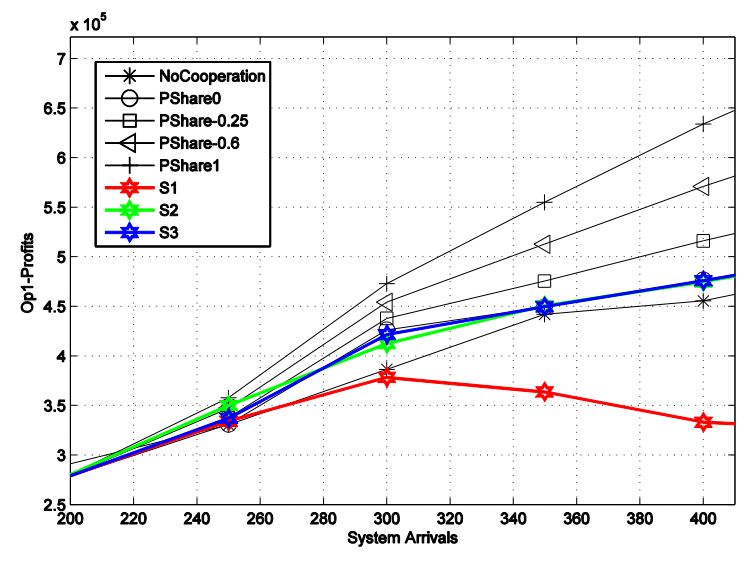

Fig. 5a. MII-Op1's achieved profits

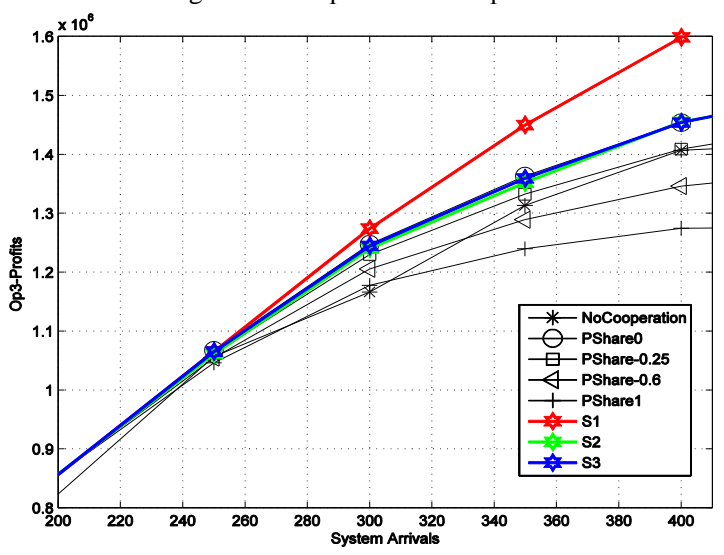

Fig. 5b.MII-Op3's achieved profits

[4] K. Johansson, Cost Effective Deployment Strategies for Heterogenous Wireless Networks : Doctoral dissertation in telecommunication, Stockholm, November 2007.

[5] L.Giupponi, R. Agusti, J. Pérez-Romeo and O. Sallent, "Improved revenue and radio resource usage through inter-operator joint radio resource management," in Proc. IEEE ICC'07, Glasgow, Scotland June 2007.

[6] L.Giupponi, R. Agusti, J. Pérez-Romeo and O. Sallent, "Inter-operator agreements based on QoS metrics for improved revenue and spectrum efficinecy", Electronics Letters Vol.44 No.4, February 2008.

[7] A. Checko, H. L. Christiansen, Y. Yan, L. Scolaris, G. Kardaras, M. S. Berger and L. Dittman "Cloud RAN for mobile networks-a technology overview", IEEE Communications surveys \& tutorials , Issue 99 , DOI 10.1109/COMST.2014.2355255

[8] X.Cao, H. Shen, R. Miloto and P. Wirth, "Internet Pricing with Game Theoretical Approach: Concepts and Examples", IEEE/ ACM Transactions on Networking, Vol. 10, April 2002.

[9] J. Elias, F. Martignon, L. Chen and E. Altman, "Joint Operator Pricing and Network Selection Game in Cognitive Radio Networks: Equilibrium, System Dynamics and Price of Anarchy", IEEE Transaction on Vehicular Technology, vol. 62, No. 9, November 2013.

[10] V. Gajié, J.Huang and B. Rimoldi, "Competition of Wireless Providers for Atomic Users", IEEE/ACM Transactions on Networking ,2013.

[11] S. Farhat, A. Samhat and S. Lahoud, "Hybrid Decision Algorithm for Access Selection in Multi-operator Networks", in Proc. IEEE WCNC'14, Istanbul, Turkey, April 2014.

[12] S. Farhat, A. Samhat, S. Lahoud and B. Cousin,"Best operator policy in a heterogeneous wireless network", in SDIWC Proc. ICeND' 14, Beirut, Lebanon, April-May 2014.

[13] J. Markendahl, A. Ghanbari, and B.G. Molleryd, "Network Cooperation Between Mobile Operators-Why and How Competitors Cooperate?", IMP conf, Atlanta 2013. 
[14] J. Hultell, K. Johansson and J. Markendahl, "Business models and resource management for shared wireless networks", in Proc. IEEE VTC'04, Los Angeles, CA, USA, September 2004. 